\title{
Stomatocyte in Stomatocyte: A New Shape of Polymersome Induced via Chemical-Addition Methodology
}

\author{
Yongjun Men, Wei Li, Geert-Jan Janssen, Roger S. M. Rikken, and Daniela A. Wilson*(i) \\ Radboud University Nijmegen, Institute for Molecules and Materials, Heyendaalseweg 135, 6525 AJ Nijmegen, The Netherlands
}

Supporting Information

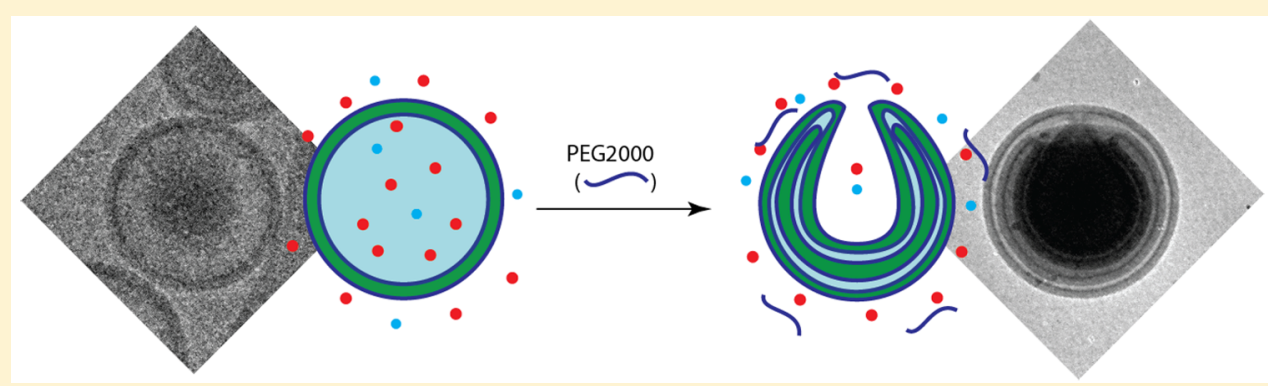

ABSTRACT: Accurate control of the shape transformation of polymersome is an important and interesting challenge that spans across disciplines such as nanomedicine and nanomachine. Here, we report a fast and facile methodology of shape manipulation of polymersome via out-of-equilibrium polymer self-assembly and shape change by chemical addition of additives. Due to its increased permeability, hydrophilicity, and fusogenic properties, poly(ethylene oxide) was selected as the additive for bringing the system out of equilibrium via fast addition into the polymersome organic solution. A new shape, stomatocyte-in-stomatocyte (sto-in-sto), is obtained for the first time. Moreover, fast shape transformation within less than 1 min to other relevant shapes such as stomatocyte and large compound vesicles was also obtained and accurately controlled in a uniform dispersion. This methodology is demonstrated as a general strategy with which to push the assembly further out of equilibrium to generate unusual nanostructures in a controllable and fast manner.

KEYWORDS: Out-of-equilibrium, self-assembly, polymersome, shape transformation, stomatocyte, stomatocyte-in-stomatocyte

$\mathrm{T}$ he ability to kinetically manipulate the shape of polymersomes, spherical vesicles assembled from diblock copolymers, into new shape morphologies has become increasingly important for the understanding of origin of cell, disease pathology, nanomedicine, and nanorobot applications. $^{1-7}$ Simpler systems were designed to gain knowledge on the adaptability of the original cell to the environment and require a mimic of the shape change of cell membrane stimulated from chemicals present in solution. ${ }^{8-20}$ Mimetic systems are also important in understanding some diseases originating from cell lesions and the key factors influencing the shape change, e.g., the shape change of human red blood cells from a biconcave discoid shape to stomatocyte or other shapes, resulting in blood diseases such as malaria or drepanocytosis. $^{21,22}$ Furthermore, the interaction between cells and vesicles depends on the shapes of the latter, which involves shapecontrolled functional drug carriers for certain diseases. ${ }^{23}$ Finally, the fabrication of biomimetic robotic devices with unusual shapes, such as active particles in stomatocyte, a bowl-shaped polymersome, requires kinetic shape-controlled procedures. ${ }^{24}$

To address this need to provide strict control over the surface area and inner volume of polymersome, methods have been developed to tune the inner volume of polymersome. This has been achieved by adjusting the external osmotic pressure of freely suspended polymersomes with flexible membrane via dialysis or solvent exchange, such that an osmotic imbalance would induce volume change and molecule rearrangement in the membrane. ${ }^{3,25}$ The best candidates for such transformations were polymersomes assembled from amphiphilic block copolymers that contained flexible membranes with reduced permeability, such that they behave like lipid vesicles (liposomes) but with additionally enhanced toughness. ${ }^{13}$ Such polymersomes, however, contained mostly flexible membranes and lacked enough toughness to retain the morphology of intermediate states during the shape transformation such as tube, stomatocytes, starfish, etc., thus limiting the number of shapes tailored from polymersome.

To precisely control these intermediate shapes, polymersomes require both a rigid membrane to keep the structure unchangeable and, upon stimuli, a membrane that is flexible enough for shape change. To solve this contradictory problem, a novel strategy was found by using organic solvent as plasticizing agent to soften the rigid membrane and capture the intermediate structures via a quenching process in water. One model system found was polymersomes assembled from poly(ethylene glycol)-block-polystyrene (PEG- $b$-PS), in which

Received: January 14, 2018

Revised: February 2, 2018

Published: February 7, 2018 
THF and dioxane were used as organic solvent to soften the PS membrane for a possibility of shape change and to tune the osmotic pressure over the membrane for inducing the shape transformation. Until now, four methodologies (dialysis against water or salt solution, reverse dialysis against solvent, solvent addition, and out-of-equilibrium control-setting an osmotic pressure difference during sample preparation followed by days of equilibration time) have been developed to tune the shapes into disc, prolate spheroids, rods, or stomatocytes based on this model system as well as new applications by encapsulation of active catalysts (platinum particles or enzymes) inside the inner compartment, resulting in autonomous movement of the particles. $^{24,26-32}$ However, all morphologies obtained with these methods are currently very limited in number, and the techniques used are laborious and time-consuming ( $>1$ day), while the system is not dynamic like giant liposomes and does not allow the generation of new exotic morphologies. ${ }^{33-35}$

Here, we propose a completely new route and methodology with which to further push the polymersome assembly out of equilibrium beyond its stomatocyte shape to reach new exotic morphologies by inducing the shape change continuously in a more-dynamic way, combining both osmotic change and controlled fusion procedure via chemical addition. We employed polyethylene glycol (PEG), to push further the polymersome transformation beyond low-energy-state stomatocyte to obtain other shapes. PEG is commonly used as a fusogen in the production of somatic hybrids and liposomes due to its dehydration effect on lipid membranes. ${ }^{36-39}$ Interestingly, we found that in this stratagem trace amount (0.005 wt \%) of PEG could successfully induced the shape change of polymersomes instead of $>15$ wt \% for the liposome system, and this strategy can not only lead to a new pathway for reaching novel structures, such as nanosized stomatocyte-instomatocyte (sto-in-sto), but also shorten the fabrication time of all of the desired shapes among which stomatocytes to less than $1 \mathrm{~min}$. Here we will mainly focus on the unusual new morphology sto-in-sto structure, its unique properties, and conditions of formation while giving a detailed description of the mechanism of formation.

The fabrication procedure of sto-in-sto shape was carried in a simple and fast manner, as illustrated in Figure 1. Spherical polymersomes were assembled from $10 \mathrm{mg}$ of $\mathrm{PEG}_{45}-\mathrm{g}_{\mathrm{g}} \mathrm{PS}_{230}$ in $1 \mathrm{~mL}$ THF $/$ dioxane $=4: 1(\mathrm{v} / \mathrm{v})$ via the slow addition of water at a rate of $1 \mathrm{~mL} / \mathrm{h} .{ }^{40}$ When the volume of water reached 0.3 $\mathrm{mL}$, just beyond the critical water point $(0.25 \mathrm{~mL}), 200 \mu \mathrm{L}$ of the polymersome suspension was transferred to a centrifuge tube. Next, $10 \mu \mathrm{L}$ of PEG2000 aqueous solution $(100 \mathrm{~g} / \mathrm{L})$ was added into the suspension $(200 \mu \mathrm{L})$ at once for a final concentration of $5 \mathrm{~g} / \mathrm{L}$. Due to the fast shape-transition procedure from this method, after the addition of PEG2000, the sample was only shaken for 1 min for solution equilibration and adequate mixing. At this state, the polymersome membrane is extremely flexible due to the high organic-solvent ratio (77 vol \%) contained, which facilitates the shape transformation.

Dry and cryo-TEM were employed to monitor the morphology change of the polymersome. Before the addition of PEG2000, the polymersome kept a thermodynamic stable spherical shape, as shown in Figures $\mathrm{S} 1$ and 2A. After the addition of PEG2000, the spherical polymersomes in a size range of $300-500 \mathrm{~nm}$ transformed into sto-in-sto shape (Figure 2C,D). This shape is difficult to generate when the vesicle size is less than $300 \mathrm{~nm}$ due to the limited cavity space. Because four layers of membrane contained in one vesicle as

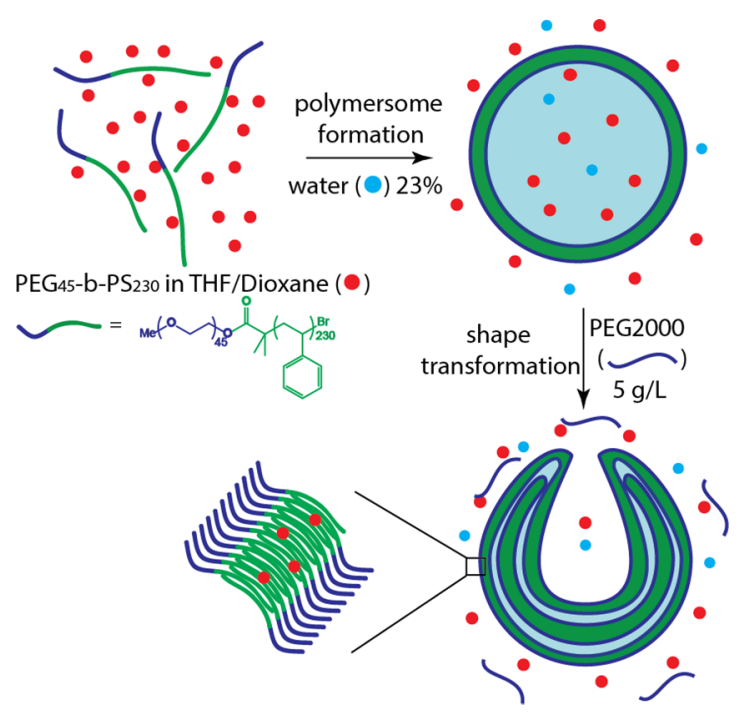

Figure 1. Schematic outlining of the chemical addition methodology induced shape transformation of spherical polymersome $\left(\mathrm{PEG}_{45^{-}} \mathrm{g}\right.$ $\mathrm{PS}_{230}$ ) into sto-in-sto shape.
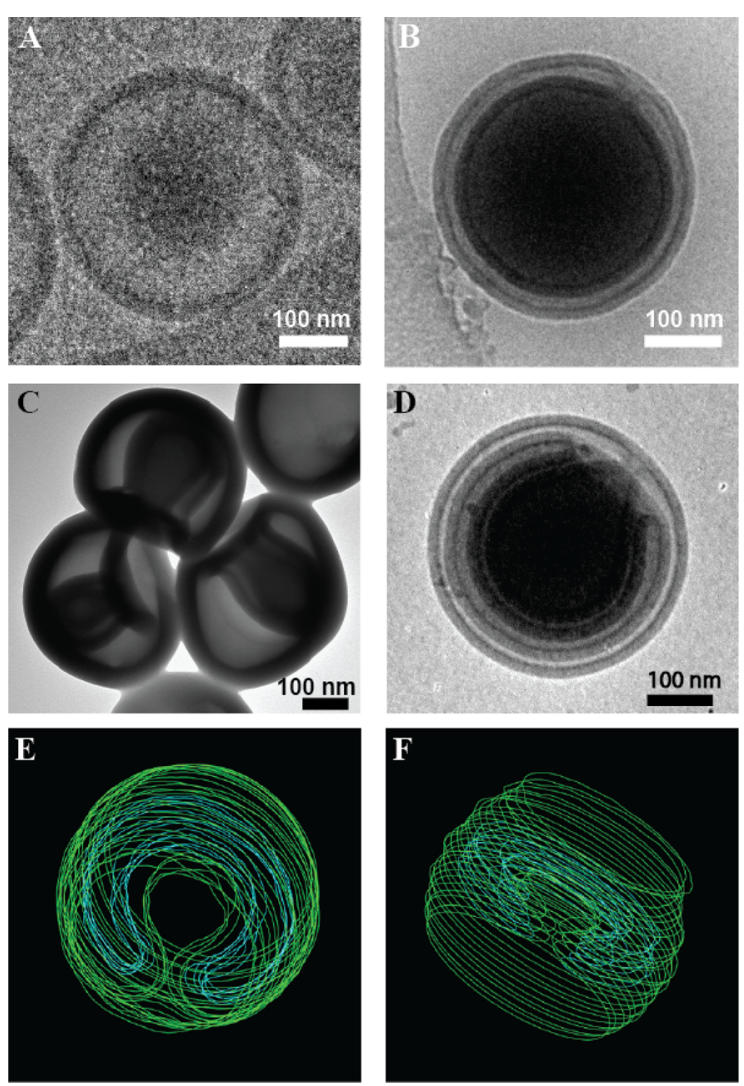

Figure 2. (A) Cryo-TEM image of polymersomes of $\mathrm{PEG}_{45}-b-\mathrm{PS}_{230}$ without shape change obtained by the rapid quenching of the suspension in water. Cryo-TEM image (B) of stomatocyte, (C) dry TEM, and (D) cryo-TEM images of stomatocytes-in-stomatocytes after shape transformation of polymersomes of $\mathrm{PEG}_{45}-b-\mathrm{PS}_{230}$, showing different projections of the structures. (E) Horizontal and (F) vertical views of $3 \mathrm{D}$ electron reconstruction of the sto-in-sto shape.

shown in Figure 2c, the average size decreased from $475 \mathrm{~nm}$ (sphere) to $361 \mathrm{~nm}$ (sto-in-sto) (by accounting for 66 particles in TEM images; Figure S2). The cyro-TEM image (Figure 2D) 
shows detailed information on this structure, with only one stomatocyte connecting to the outer environment, while the other stomatocyte inserts between the two membranes of the outer one. The thickness of the membrane was measured from cryo-TEM image, with an average of $20.5 \pm 1 \mathrm{~nm}$ (Figure S3), which is the same as spherical shape, suggesting that the polymer chains are arranged in the same manner as during the shape change. Moreover, the membrane thicknesses are the same from both outer and inner stomatocytes. To gain structural detail and proof of the inner structure of the selfassembled shape, 3D electro tomography, which allows for successive tilt acquisition and $3 \mathrm{D}$ reconstruction of the sto-insto polymersome, was constructed (Figure 2E,F and Video S1). The results further demonstrate that the open mouth of the sto-in-sto polymersome is very small, only around $19 \pm 3 \mathrm{~nm}$, and the inner stomatocyte is inserted evenly between the membranes of the outer stomatocyte.

To form this unique sto-in-sto structure, PEG indeed has a direct major contribution in this process. As previously known, the shape change of polymersome is dominantly controlled by osmotic pressure differences between the inner and outer of the vesicle. PEG is a polyether composed of ethylene oxide, and each ethylene oxide unit could bind two or three water molecules, ${ }^{41}$ which possibly creates different osmotic gradients. $^{42,43}$ Therefore, we studied different amounts of PEG as the additive to test the shape change. As presented in Figure 3,
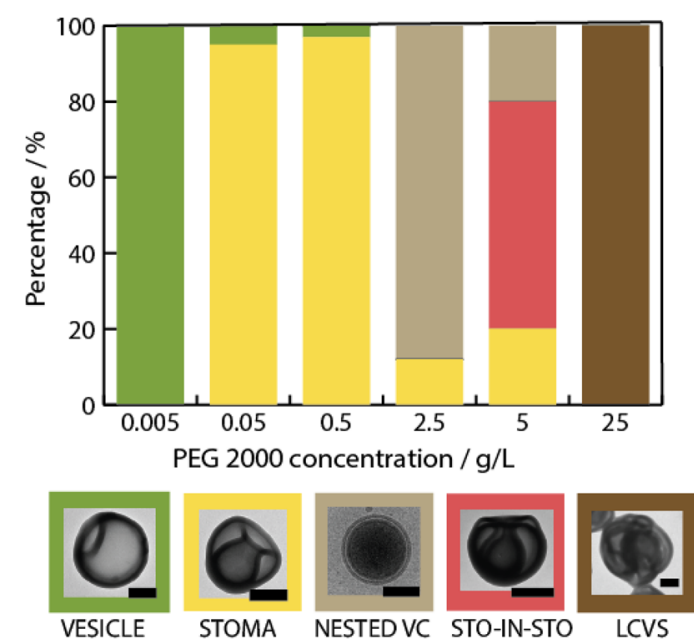

Figure 3. Statistical evaluation of polymersome morphologies induced by addition of different amounts of PEG2000. STOMA: stomatocytes, NESTED VC: nested vesicles, STO-IN-STO: stomatocyte-instomatocyte, LCVS: large component vesicles. Scale bar: $200 \mathrm{~nm}$.

the addition of very small amounts $(0.005 \mathrm{~g} / \mathrm{L})$ of PEG2000 has no influence on the shape of the polymersomes, the structures maintaining their spherical morphology (Figure S4A). Increasing PEG2000 concentration from 0.05 to $0.5 \mathrm{~g} /$ $\mathrm{L}$, stomatocyte (mono- and multiopened) could be obtained (Figure S4B,C). This transition was known as osmotic pressure induced change. ${ }^{26}$ It is worth noting that this PEG addition process was the simplest and fastest methodology found to fabricate large-scale uniform stomatocyte until now. ${ }^{24,26,28-30}$ Once the concentration of PEG2000 reached higher values above $5 \mathrm{~g} / \mathrm{L}$, an unusual mixture of structures with shape polydispersity was obtained, ranging from stomatocytes, nested vesicles and sto-in-sto structures (Figure S4E); the ratio was accounted for by a calculation of TEM images. One reason to form such multiple structures might be the ability of PEG2000 in high concentrations to induce fusion of polymersomes, with the same fusogenic properties as in liposome due to involving short-range forces critically dependent on the destabilization of the bilayers by dehydration and structural water. ${ }^{36-39,44}$ Increasing the PEG2000 concentration to $25 \mathrm{~g} / \mathrm{L}$, the membranes of polymersomes are all fused together and aggregated to form large-component vesicle (LCVs) (Figure S4F), which further demonstrated the fusogenic properties of PEG2000, caused by PEG adsorption and depletion, being affected by many factors, one of which is the vesicle surface properties.

The capability of PEG to act as fusogen of polymersome membrane as well as strong inducer of osmotic pressure change is expected to be strongly influenced by its molecular weight. For example, small PEG at a low concentration has less ability to induce osmotic pressure differences over the membrane. Therefore, besides PEG2000, we chose PEG400 and PEG10000 to perform experiments under the same conditions for preparing sto-in-sto structures. Equal-weight amounts of these PEG polymers were used to keep the same number of ethylene oxide unit that has the capability to bind equal amount of water molecules. As expected, when using a short polymer length of PEG400, no effect on the shape of polymersomes was observed (Figure S5) even at high concentrations of $25 \mathrm{~g} / \mathrm{L}$. This is most probably due to a lack of depletion effect and the lower ability of bonding water. Large0size PEG10000 molecules perform a similar effect as did PEG2000 (Figure S6) based on the "osmophobic association" theory. Sto-in-sto shape was obtained at a concentration of $5 \mathrm{~g} / \mathrm{L}$, and at $25 \mathrm{~g} / \mathrm{L}$, LCVs were formed, indicating that PEG10000 has similar fusogenic properties as PEG2000 on this polymersome. However, lower concentrations of the additive between 0.005 and $2.5 \mathrm{~g} / \mathrm{L}$, PEG10000 performed higher shape-induce ability. As shown in the TEM images, at $0.005 \mathrm{~g} / \mathrm{L}$ about $40 \%$ of polymersomes changed to stomatocytes compared to no stomatocyte observed with PEG2000 at the same conditions; at $0.05 \mathrm{~g} / \mathrm{L}$, a tubule shape was captured; at $0.5 \mathrm{~g} / \mathrm{L}$, a well-shaped stomatocyte formed; at $2.5 \mathrm{~g} / \mathrm{L}$, mainly nested vesicles were obtained. These results indicate that large molecular weights of PEG have stronger capability for the shape transformation of polymersome.

Based on the above results and analysis, a pathway associated with osmotic pressure guiding and inner fusion process can be suspected for formation of sto-in-sto, which makes this method generally applicable to other amphiphilic block copolymers. We should mention here that the shape-transition procedure of polymersome is discontinued and too fast to be captured; thus, the proofs of the suspected pathway are only based on the collected intermediate structures from TEM images and former reports. As demonstrated from calculation and experimental evidence reported before, polymersome prefers to follow a lowenergy route to achieve the disc and stomatocyte shapes, ${ }^{28,45}$ coinciding with the fact that stomatocytes are the major shapes when PEG2000 concentrations are $0.05-0.5 \mathrm{~g} / \mathrm{L}$. Thus, we believe that the stomatocyte shape is mainly guided by osmotic pressure change. A high concentration of PEG2000 facilitates membrane fusion to obtain nested vesicles morphology due to the osmotic induction of the high membrane-curvature promotion, $^{38}$ and the PEG2000 induced a further shape change to the sto-in-sto through a disc-in-disc (suspected) intermediary structure (Figure 4). 


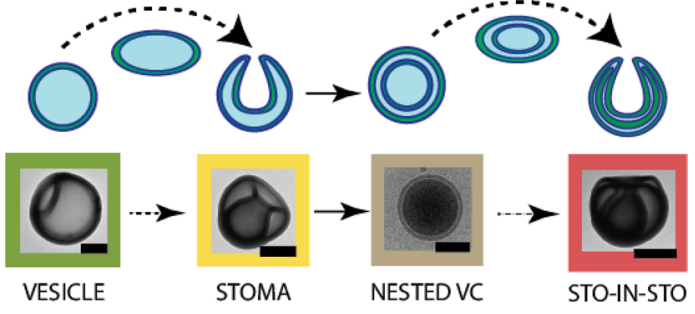

Figure 4. Suspected pathway of sto-in-sto formation of polymersomes. A spherical polymersome transferred to disc and stomatocyte shape. Next, the mouth of stomatocyte fused to the nest structure, followed with a deflation to disc-in-disc, and bent to sto-in-sto at last. Note: disc and disc-in-disc shapes are suspected as intermediate shapes, which were not captured in this experiment due to the fast transition procedure. Scale bar: $200 \mathrm{~nm}$.

In conclusion, we demonstrate for the first time the folding of a self-assembled polymersome in the sto-in-sto unique morphology. We achieve this by bringing the supramolecular assembly out of equilibrium via the chemical addition of PEG fusogen to further push the polymersome transformation beyond low-energy-state stomatocyte to this unusual shape, further reaching to a thermodynamic LCVs state. ${ }^{46}$ Cryo-TEM and $3 \mathrm{D}$ reconstruction provided a detailed image showing that the inner stomatocyte is located in the cavity of the outer one. We found that even traces amounts of PEG (0.005 wt \%) are sufficient to induce the shape change of the polymersomes, resulting in not only a new methodology for controlled shapes but also a new pathway for reaching novel structures, such as nanosized stomatocyte-in-stomatocyte. Furthermore, this new methodology significantly reduces the fabrication time of all of the desired shapes including stomatocytes to less than $1 \mathrm{~min}$ and is expected to be suitable to a wide range of polymersomes. This is particularly important because the formation of stomatocytes was previously requiring time-consuming methods. PEG was demonstrated as a crucial factor to induce the shape change due to its binding water property for inducing osmotic pressure change and its fusogenic ability. We believe that this strategy could provide a new platform for shape control and the assembly of wider polymeric nanostructures with unusual architectures and functions.

\section{ASSOCIATED CONTENT}

\section{S Supporting Information}

The Supporting Information is available free of charge on the ACS Publications website at DOI: 10.1021/acs.nanolett.8b00187.

Additional experimental details. Figures showing TEM images, calculation of size and distribution, and membrane thickness. (PDF)

A video showing $3 \mathrm{D}$ reconstruction of the sto-in-sto polymersome. (AVI)

\section{AUTHOR INFORMATION}

\section{Corresponding Author}

*E-mail: d.wilson@science.ru.nl. Phone: +312-436-52185. Fax: +312-436-53450.

\section{ORCID $\odot$}

Daniela A. Wilson: 0000-0002-8796-2274

\section{Author Contributions}

Y.M. and D.A.W. designed the experiment and wrote the manuscript. Y.M., W.L., and R.S.M.R. performed the experiments. G.-J.J. performed the cryo-TEM measurement and 3D reconstruction.

\section{Funding}

The authors acknowledges the European Research Council ERC-StG 307679 "StomaMotors" and NWO Chemiche Wetenschappen VIDI, grant no. 723.015.001, for financial support. We acknowledge support from the Ministry of Education, Culture and Science (gravitation program 024.001.035).

\section{Notes}

The authors declare no competing financial interest.

\section{ACKNOWLEDGMENTS}

Y.M. acknowledges fruitful discussion with Prof. Jan van Hest and Prof. Roeland Nolte.

\section{ABBREVIATIONS}

STOMA, stomatocytes; NESTED VC, nested vesicles; STOIN-STO, stomatocyte-in-stomatocyte; LCVS, large-component vesicles

\section{REFERENCES}

(1) de Oliveira, H.; Sandre, O.; Pourtau, L.; Andrés, E.; Miraux, S.; Thiaudière, E.; Berra, E.; Lecommandoux, S.; Thevenot, J. J. Controlled Release 2013, 172, e44-e45.

(2) Srinivas, G.; Discher, D. E.; Klein, M. L. Nat. Mater. 2004, 3, 638-644.

(3) Salva, R.; Le Meins, J.-F.; Sandre, O.; Brûlet, A.; Schmutz, M.; Guenoun, P.; Lecommandoux, S. ACS Nano 2013, 7, 9298-9311.

(4) Wang, L.; Liu, G.; Wang, X.; Hu, J.; Zhang, G.; Liu, S. Macromolecules 2015, 48, 7262-7272.

(5) Robertson, J. D.; Yealland, G.; Avila-Olias, M.; Chierico, L.; Bandmann, O.; Renshaw, S. A.; Battaglia, G. ACS Nano 2014, 8, 4650-4661.

(6) Deng, Z.; Qian, Y.; Yu, Y.; Liu, G.; Hu, J.; Zhang, G.; Liu, S. J. Am. Chem. Soc. 2016, 138, 10452-10466.

(7) Zhu, J.; Zhang, S.; Zhang, K.; Wang, X.; Mays, J. W.; Wooley, K. L.; Pochan, D. J. Nat. Commun. 2013, 4, 2297-2305.

(8) van Hest, J. C. M.; Delnoye, D. A. P.; Baars, M. W. P. L.; van Genderen, M. H. P.; Meijer, E. W. Science 1995, 268, 1592-1595.

(9) Zhang, L.; Eisenberg, A. Science 1995, 268, 1728-1731.

(10) Zhang, L.; Eisenberg, A. Macromolecules 1996, 29, 8805-8815.

(11) Zhang, L.; Eisenberg, A. J. Am. Chem. Soc. 1996, 118, 31683181.

(12) Zhang, L.; Yu, K.; Eisenberg, A. Science 1996, 272, 1777-1779.

(13) Discher, B. M.; Won, Y.-Y.; Ege, D. S.; Lee, J. C.-M.; Bates, F. S.; Discher, D. E.; Hammer, D. A. Science 1999, 284, 1143-1146.

(14) Bermudez, H.; Brannan, A. K.; Hammer, D. A.; Bates, F. S.; Discher, D. E. Macromolecules 2002, 35, 8203-8208.

(15) Pochan, D. J.; Chen, Z.; Cui, H.; Hales, K.; Qi, K.; Wooley, K. L. Science 2004, 306, 94-97.

(16) Du, J.; Tang, Y.; Lewis, A. L.; Armes, S. P. J. Am. Chem. Soc. 2005, 127, 17982-17983.

(17) Kita-Tokarczyk, K.; Grumelard, J.; Haefele, T.; Meier, W. Polymer 2005, 46, 3540-3563.

(18) Ruysschaert, T.; Sonnen, A. F. P.; Haefele, T.; Meier, W.; Winterhalter, M.; Fournier, D. J. Am. Chem. Soc. 2005, 127, 62426247.

(19) Ahmed, F.; Photos, P. J.; Discher, D. E. Drug Dev. Res. 2006, 67, $4-14$.

(20) Zong, W.; Ma, S.; Zhang, X.; Wang, X.; Li, Q.; Han, X. J. Am. Chem. Soc. 2017, 139, 9955-9960.

(21) Uzoigwe, C. Med. Hypotheses 2006, 67, 1159-1163. 
(22) Doshi, N.; Zahr, A. S.; Bhaskar, S.; Lahann, J.; Mitragotri, S.

Proc. Natl. Acad. Sci. U. S. A. 2009, 106, 21495-21499.

(23) van der Weijden, J.; Paulis, L. E.; Verdoes, M.; van Hest, J. C. M.; Figdor, C. G. Chem. Sci. 2014, 5, 3355-3367.

(24) Wilson, D. A.; Nolte, R. J.; van Hest, J. C. Nat. Chem. 2012, 4, 268-274.

(25) Yan, Q.; Wang, J.; Yin, Y.; Yuan, J. Angew. Chem., Int. Ed. 2013, 52, 5070-5073.

(26) Kim, K. T.; Zhu, J.; Meeuwissen, S. A.; Cornelissen, J. J. L. M.; Pochan, D. J.; Nolte, R. J. M.; van Hest, J. C. M. J. Am. Chem. Soc. 2010, 132, 12522-12524.

(27) Abdelmohsen, L. K. E. A.; Williams, D. S.; Pille, J.; Ozel, S. G.; Rikken, R. S. M.; Wilson, D. A.; van Hest, J. C. M. J. Am. Chem. Soc. 2016, 138, 9353-9356.

(28) Rikken, R. S. M.; Engelkamp, H.; Nolte, R. J. M.; Maan, J. C.; van Hest, J. C. M.; Wilson, D. A.; Christianen, P. C. M. Nat. Commun. 2016, 7, 12606-12611.

(29) Abdelmohsen, L. K. E. A.; Nijemeisland, M.; Pawar, G. M.; Janssen, G.-J. A.; Nolte, R. J. M.; van Hest, J. C. M.; Wilson, D. A. ACS Nano 2016, 10, 2652-2660.

(30) Meeuwissen, S. A.; Kim, K. T.; Chen, Y.; Pochan, D. J.; van Hest, J. C. M. Angew. Chem., Int. Ed. 2011, 50, 7070-7073.

(31) Tu, Y.; Peng, F.; André, A. A. M.; Men, Y.; Srinivas, M.; Wilson, D. A. ACS Nano 2017, 11, 1957-1963.

(32) Tu, Y.; Peng, F.; Sui, X.; Men, Y.; White, P. B.; van Hest, J. C. M.; Wilson, D. A. Nat. Chem. 2016, 9, 480-486.

(33) Berndl, K.; Käs, J.; Lipowsky, R.; Sackmann, E.; Seifert, U. EPL (Europhysics Letters) 1990, 13, 659.

(34) Noguchi, H. EPL (Europhysics Letters) 2015, 112, 58004.

(35) Hotani, H. J. Mol. Biol. 1984, 178, 113-120.

(36) Lee, J.; Lentz, B. R. Biochemistry 1997, 36, 421-431.

(37) Lehtonen, J. Y.; Kinnunen, P. K. Biophys. J. 1994, 66, 19811990.

(38) Malinin, V. S.; Frederik, P.; Lentz, B. R. Biophys. J. 2002, 82, 2090-2100.

(39) Käsbauer, M.; Lasic, D. D.; Winterhalter, M. Chem. Phys. Lipids 1997, 86, 153-159.

(40) Men, Y.; Peng, F.; Tu, Y.; van Hest, J. C. M.; Wilson, D. A. Polym. Chem. 2016, 7, 3977-3982.

(41) Graham, N. B.; Zulfiqar, M.; Nwachuku, N. E.; Rashid, A. Polymer 1989, 30, 528-533.

(42) Burgess, S. W.; Massenburg, D.; Yates, J.; Lentz, B. R. Biochemistry 1991, 30, 4193-4200.

(43) MacDonald, R. I. Biochemistry 1985, 24, 4058-4066.

(44) Dong, S.; Leng, J.; Feng, Y.; Liu, M.; Stackhouse, C. J.; Schönhals, A.; Chiappisi, L.; Gao, L.; Chen, W.; Shang, J.; Jin, L.; Qi, Z.; Schalley, C. A. Sci. Adv. 2017, 3, 1 DOI: 10.1126/sciadv.aao0900.

(45) Rikken, R. S. M.; Kerkenaar, H. H. M.; Nolte, R. J. M.; Maan, J. C.; van Hest, J. C. M.; Christianen, P. C. M.; Wilson, D. A. Chem. Commun. 2014, 50, 5394-5396.

(46) Wang, J.; Liu, K.; Xing, R.; Yan, X. Chem. Soc. Rev. 2016, 45, 5589-5604. 